A4

doi: 10.14232/ syrpharmacognosy.2021.a4

\title{
Isolation and structure determination of compounds from Juncus articulatus
}

Anita Barta

Email: bartaanita96@gmail.com

Juncaceae species are characterized by the synthesis and accumulation of phenanthrenes. These compounds are of great interest from structural, pharmacological, semisynthetic and chemotaxonomical points of view. The aim of our work is the phytochemical and pharmacological investigation of Juncaceae species occurring in the Carpathian Basin. In the course of this work, the investigation of the secondary metabolites of J. articulatus, a perennial plant grows in moist areas, such as wet sand, is performed [1,2].

The dried and ground plant material was extracted with methanol. After concentration, the extract was dissolved in $50 \%$ aqueous methanol, and solvent-solvent partitions were performed with $n$-hexane, chloroform and ethyl acetate. Based on the preliminary studies, it was concluded that phenanthrenes are enriched in the chloroform phase. Therefore, at first this fraction was separated by column chromatography and then further purified by Sephadex LH-20 gel chromatography. As a final purification, preparative thin-layer chromatography and highperformance liquid chromatography are used. Structure elucidation of the isolated compounds is carried out by NMR and MS spectroscopy as well as by comparison with literature data.

To date, three compounds were identified, two phenanthrenes and one flavonoid.

Supervisor: Andrea Vasas

\section{Acknowledgements:}

This work was supported by National Research, Development and Innovation Office, Hungary (NKFIH; K128963).

\section{References}

[1] Tóth B, Hohmann J, Vasas A. J. Nat. Prod. 2018; 81: 661-678.

[2] Bús Cs, et al. Phytochem. Rev. 2018; 17: 833-851 